\title{
Viral Respiratory Infections in Children Receiving Chemotherapy or Undergoing Stem Cell Transplantation
}

\section{Christianna Vliora $^{1 *}$, Garyfallia Syridou ${ }^{2}$ and Vassiliki Papaevangelou ${ }^{1}$}

${ }^{1}$ Third Department of Pediatrics, National and Kapodistrian, University of Athens, Athens, Greece

${ }^{2}$ Second Department of Pediatrics, National and Kapodistrian, University of Athens, Athens, Greece

\begin{abstract}
In immunocompromised hematology/oncology patients, respiratory infections are both common and potentially severe. However, there is still a noticeable gap in the medical literature, regarding the epidemiology of viral respiratory infections in pediatric hematology/oncology patients. This is mainly due to research being focused on readily treatable causes and to limitations by time-consuming diagnostic techniques. Recent developments, though, have produced sensitive and accurate methods for detecting viruses, yielding results within hours and able to screen for multiple species and subtypes, illuminating at the same time "novel" viruses that cause disease in immunocompromised patients. In this review, the epidemiology and disease burden of viral respiratory infections among pediatric oncology patients and hemopoietic stem cell transplantation recipients are discussed. Additionally, recent therapeutic and preventive options promising improved outcomes are reviewed.
\end{abstract}

Keywords: Oncology; Chemotherapy; Children; Respiratory Viral Infections

\begin{abstract}
Abbreviations: HSCT: Hematopoietic Stem Cell Transplantations; ALL: Acute Lymphoblastic Leukemia; URI: Upper Respiratory Infections; Hmpv: Human Metapneumovirus; RSV: Respiratory Syncytial Virus; AML: Acute Myeloblastic Leukemia; LRI: Lower Respiratory Infections; CMV: Cytomegalovirus; Gvhd: Graft Versus Host Disease; BAL: Bronchoalveolar Lavage; PICU: Pediatric Intensive Care Unit; IVIG: Intravenous Immunoglobulin
\end{abstract}

\section{Introduction}

In paediatric oncology patients and those receiving HSCT, infections cause significant morbidity and mortality [1]. Although for decades, bacterial and fungal infections have been the focus of research, recently improved diagnostic capabilities have revealed that viral pathogens are also associated with an increased morbidity and mortality $[2,3]$. Viral respiratory tract infections are common and may present with a wide range of clinical syndromes from asymptomatic infection to severe pneumonia and death $[4,5]$. In a pediatric ALL study, $43.5 \%$ of all infectious episodes were respiratory, of which $88.5 \%$ represented URI [6]. In addition viral respiratory infections in children with HSCT have been associated with mortality ranging from $10 \%$ to $14 \%[7,8]$. Research on viruses has been limited by the use of lengthy and complicated techniques, such as viral culture, infected tissue biopsy and serology. The past decades, though, have witnessed studies utilizing molecular methods (PCR/RT-PCR) to determine the epidemiology of respiratory viruses in adult and paediatric cancer patients. These methods have significantly increased the sensitivity of detection and filled the diagnostic gap in cancer patients with either respiratory infections or with "fever of unknown origin" [9]. Moreover, "novel" pathogens, such as hMPV, bocavirus have been identified and seem to play an important role in immunocompromised cancer patients $[4,10$ 12].

Infections of the upper and lower respiratory tract are caused by a number of viruses, mainly influenza A and B, parainfluenza 1-4, RSV, adenovirus, rhinovirus, hMPV and boca-virus (Table 1). The prevalence of viral respiratory infections depends on the geographic area, season, exposure, virulence, types of circulating viruses and influenced by the detection method used [11]. Often, immunosuppressed patients act as outbreak "sentinels" [11]. Distinction amongst the various respiratory viruses based on clinical symptoms and signs, is not feasible, since clinical presentation is similar. Immunocompromised children may display mild nonspecific signs and symptoms or may exhibit either a prolonged duration of febrile illness or a rapidly deteriorating disease.

\section{Epidemiology}

\section{Hematologic malignancies}

Two surveys to date, have utilized molecular methods (PCR/ RT-PCR) to determine respiratory viruses epidemiology in pediatric leukemia patients $[13,14]$. In a prospective study of febrile leukemic children, respiratory viruses were detected in $44 \%$, with rhinovirus (22\%) and RSV (11\%) being the most prevalent. Respiratory infections were usually mild with only two cases $(3.2 \%)$ progressing to LRI. Lymphopenia was the only risk factor associated with virus acquisition. However it must be mentioned that the choice to use oral washes in this study, may account for the diminished viral yield of the samples [14]. In an earlier study of children with cancer, a viral pathogen was found in only $11 \%$, again with rhinovirus and RSV being the most frequent agents identified. Interestingly, in this cohort $74 \%$ of children were neutropenic [13]. These findings are in accordance with an earlier study, utilizing standard methods (serology etc), where rhinovirus was also the predominant isolate (17\%), with RSV and parainfluenza 3 following [15].

The contribution of chemotherapy intensity, on the epidemiology

*Corresponding author: Christianna Vliora, Third Department of Pediatrics, Univerity of Athens, Attikon, University General Hospital, Rimini 1 Chaidari, 12462, Athens, Greece, Tel: 0030-2105832228; Fax: +30-2105832229; E-mail: xristiannavliora@gmail.com

Received January 09, 2014; Accepted January 28, 2014; Published February 03, 2014

Citation: Vliora C, Syridou G, Papaevangelou V (2014) Viral Respiratory Infections in Children Receiving Chemotherapy or Undergoing Stem Cell Transplantation. Clin Microbial 3: 137. doi:10.4172/2327-5073.1000137

Copyright: (c) 2014 Vliora C, et al. This is an open-access article distributed under the terms of the Creative Commons Attribution License, which permits unrestricted use, distribution, and reproduction in any medium, provided the original author and source are credited. 


\begin{tabular}{|l|c|c|}
\hline Influenza A,B & Negative sense, single-stranded RNA orthomyxoviruses & Droplet precautions \\
\hline Parainfluenza 1-4 & Single-stranded RNA paramyxoviruses & Droplet precautions \\
\hline RSV & Single-stranded RNA paramyxoviruses & Droplet precautions \\
\hline hMPV & Negative-sense nonsegmented RNA paramyxovirus & Droplet precautions \\
\hline Rhinovirus & RNA Picornavirus & Droplet precautions \\
\hline Adenovirus & Nonenveloped, double-stranded DNA & Droplet precautions \\
\hline Bocavirus & Nonenveloped, single-stranded DNA Parvovirus & Droplet and contact precautions \\
\hline
\end{tabular}

Table 1: Characteristics and precaution measures of the most common respiratory viruses.

of viral respiratory infections has been evaluated in two pediatric studies involving children with AML and ALL respectively. The AML study, compared two groups of patients, allocated to receive either intensive or standard chemotherapy. All types of infection occurred more frequently during intensive versus standard (bacterial- $57.7 \%$ vs. $39.4 \%$, fungal- $27.4 \%$ vs. $9.9 \%$, viral- $14 \%$ vs. $3.9 \%$ ). During intensive induction, respiratory viruses' percentage consisted of influenza $(8 \%)$, parainfluenza (6\%), adenovirus (4\%), and RSV (4\%) [16]. In the ALL study, among exclusively infants, receiving a 6-phase chemotherapy protocol viral infections accounted for $13 \%$ of overall infections, second only to bacterial (74\%). Of note mortality rate was increased during induction (phase 1) (16.5\%), in contrast to $1.9 \%$ of consolidation (phase 4). During induction, viral infections were quite lethal, with 6 patients out of 23 having a fatal outcome. In particular LRI were predominantly viral in origin (39\%), caused by RSV and influenza, followed by fungal (27\%) and bacterial (20\%) [17]. Another retrospective study of paediatric AML patients focused solely on RSV, and reported mortality of $10 \%(4 / 40)$. Three of the children developed RSV-infection during induction and the fourth during intensification supporting possible association between phase of chemotherapy and outcome [18].

\section{Solid tumors}

As a whole, solid tumors represent $60 \%$ of childhood cancer, with CNS masses and sympathetic system tumors (neuroblastoma, ganglioneuroblastoma) being most prevalent [19]. Despite this, viral respiratory infections in children with solid tumors haven't been studied independently of hematologic malignancies. A recent study reported that patients with solid tumors had significantly more viral infections but seemed to tolerate them better and required shorter hospitalisation [13]. A study of influenza in a pediatric oncology subpopulation, reported that 27 out of 511 influenza-documented illnesses occurred in children with cancer and more than $1 / 3$ had been diagnosed with solid tumors [20].

\section{Hemopoietic stem cell transplantation}

Comprehensive studies, analyzing viral epidemiology and risk factors exist mainly for adults undergoing transplantation. Recent studies in different sizes adult populations have described virus-specific incidence rates for RSV (5\%), parainfluenza (7\%), adenovirus (5\%) and influenza (1-2\%) [21-24]. Adult studies have produced conflicting results in evaluating risk factors. A Seattle-based study found that the only factor for virus acquisition was recipient CMV seropositivity [25]. Nichols et al found that female sex and advanced disease were the main identifiable predictors for influenza acquisition and allogeneic patients were more likely to acquire parainfluenza-3 [21,22]. In a paediatric HSCT study, allogeneic transplantation and grade 3-4 GvHD were identified as risk factors associated with increased incidence of viral respiratory infections. Once again parainfluenza-3 was the most frequent viral isolate [7]. A number of HSCT studies focus solely on adenovirus, conveying an unclear image of the incidence, mortality and risk factors, since there is much variation in patients' age, adenovirus detection practices, conditioning used and GvHD-preventive regimens
[11]. In the study by Hale et al adenovirus was identified in 13 of 206 pediatric patients undergoing 215 bone marrow transplants, resulting in an incidence of $6 \%$. Most patients did not exhibit respiratory symptoms, while LRI development was associated with significant mortality (50\%). Identified risk factors for infection included allogeneic transplantation and total-body-irradiation conditioning [26]. In another study of allogeneic paediatric recipients, adenoviral incidence was $12 \%$, but only 1 patient died of adenovirus pneumonia. Finally immunity recovery post-HSCT (i.e. serum IgM concentrations, peripheral T lymphocytes and subsets counts) was delayed in adenovirus-infected compared with noninfected children [27]. Runde et al. found that moderate to severe GvHD correlated with higher adenovirus infection risk. This study also supports that adenovirus infections do not always represent reactivations in the recipient but may also arrive by "transfusion" of infected cells from seropositive donors [28].

\section{Disease Burden and Complications}

Viral respiratory infections are often complicated by bacterial or fungal super-infections in cancer patients; $39 \%$ of pediatric oncology patients with RSV had a concurrent bacterial infection [29]. Twenty five percent of adults and $22.7 \%$ of children with viral pneumonia had bacterial co-pathogens isolated from BAL [30]. Additionally, cancer patients are at a higher risk to develop LRI and respiratory failure. For adult HSCT patients respiratory viral infections have been reported to progress from URI to LRI in $18 \%$ to $44 \%$ of cases, depending on the population studied [21-24]. A risk factor for influenza progression to LRI seems to be autologous (47\%) versus allogeneic HSCT (31\%) [22]. Disease burden is an important issue; In a study of El Saleeby et al. 36\% of children with cancer and RSV infection required hospitalization, $22 \%$ oxygen administration, while $9 \%$ mechanical ventilation [31]. In Mendoza-Sanchez et al. study, $21 \%$ of children with respiratory tract infection receiving anticancer therapy had to be admitted to the PICU [32].

Mortality is a major concern and data refers mainly to RSV infections. In the AML- RSV study, RSV-specific mortality was $0.2 \%$ (4 out of 2,078 patients enrolled), however the risk of RSV-related mortality among RSV infections was high (10\%- 4 out of 40 patients with RSV infection), prompting discussion about the importance of appropriate management, rather than prophylaxis [18]. In the infantile- ALL study RSV mortality rate was $66 \%$ during induction versus an overall rate of $33 \%$ [17]. In another study, young age $(\leq 2$ years) and profound lymphopenia where associated with increased RSV mortality [31]. Influenza mortality seems to be associated with the development of LRI; since influenza LRI mortality was $28 \%$ in HSCT patients, significantly higher than that among subjects with URI (3\%) [22]. Overall, in adult HSCT patients mortality post viral respiratory infections is high, ranging from $25-45 \%$ [21-24]. Finally, in a study referring to children post allogeneic HSCT, the mortality rate due to viral infections was $9.7 \%$, with a respiratory virus being identified in only $20 \%$ of deaths [33].

Moreover, clinicians should also consider prolonged viral shedding. 
In a study of HSCT recipients the median duration of shedding for rhinovirus was 5 weeks (range, 1-49 weeks) [34]. The median duration of parainfluenza-3 shedding in hematologic malignancies patients was 72 days [35]. This point needs further elucidation, since asymptomatic shedding might eventually progress to symptomatic respiratory disease and may be associated with long-term decline in lung function [25]. These findings may also have implications for the role of nosocomial transmission within oncology units [25].

Finally an important consequence, when acquiring a viral infection is the delay in the chemotherapy regimen. Forty to fifty per cent of oncology patients experienced a median delay of 7 days in scheduled chemotherapy due to respiratory viral infections [20,32].

\section{Treatment}

Until recently, symptomatic therapy (adequate fluids, fever treatment, monitoring, oxygen supplementation) was the only feasible treatment option. Over the last years though, novel antiviral agents have become available, targeting specific viruses $[10,12]$.

Amongst the first antivirals used were the M2 inhibitors, amantadine and rimantadine (Table 2) [36]. Both have efficacy against Influenza A and can also be used for chemoprophylaxis. Unfortunately the emergence of resistant influenza A strains, in combination with increased toxicity of these agents have led to a decline in their use. As a result the current mainstays for influenza treatment are the neuraminidase inhibitors oseltamivir and zanamivir. This class of antivirals has the added advantage of efficacy against both Influenza A and B. In immunocompromised patients, these drugs have been shown to be effective when used in standard regimens, but also increased doses and prolonging the administration in persistent cases have been suggested [11]. Concerns have arisen, though, in cancer patients, regarding the pulmonary bioavailability of zanamivir [37]. Currently novel compounds such as peramivir are being studied in clinical trials [11].
Another antiviral drug that has been used is ribavirin, a synthetic nucleoside analog of guanosine. Ribavirin has been studied for treatment against RSV, influenza, parainfluenza, hMPV and adenovirus, mainly in adult populations. When initiated before the onset of respiratory failure in the course of uncomplicated influenza, ribavirin seems to have some benefit. However results for parainfluenza are conflicting [10]. In a HSCT study of adults with parainfluenza-3, ribavirin with or without IVIG failed to show noticeable results in lowering mortality or shortening viral shedding [21]. Regarding hMPV, ribavirin demonstrates activity both in vitro and in animal models [11]. For severe RSV, there has been some limited data on potentially beneficial effect of nebulized ribavirin for both previously healthy and immunosuppressed patients $[10,12]$. One study of RSV in adults with leukemia reported that patients in which ribavirin was not administered early (while at the URI stage), were at increased risk for progression to pneumonia (96\% vs. $68 \%$ ). Treatment with ribavirin plus IVIG lowered mortality from $36 \%$ to $6 \%$ [38]. Despite its benefit ribavirin via inhalation poses various challenges, requiring complicated and expensive techniques (continuous delivery, small particle aerosol generator unit, scavenging tent) as well as contamination prevention for healthcare workers due to its teratogenicity.

Passive immunoprophylaxis with humanised monoclonal antibodies against the F- protein of the virus (palivizumab) is not established in paediatric cancer patients [5]; in addition combination therapies of ribavirin and palivizumab have produced conflicting results. Motavizumab, an ultra-potent, affinity matured, humanised monoclonal antibody is under investigation for children with malignancies with severe RSV, as well as a RNAi molecule (ALNRSV01) that silences the nucleocapsid gene of the RSV genome [12].

Cidofovir has been used to treat severe adenovirus infections after HSCT with promising results, using various treatment regimens [39-41]. As cidofovir therapy poses major toxicity risks, mainly nephrotoxicity, adequate hydration and the co- administration of probenecid are paramount (Table 3) [42].

\begin{tabular}{|c|c|}
\hline Amantadine (Symmetrel) $)^{a}$ & 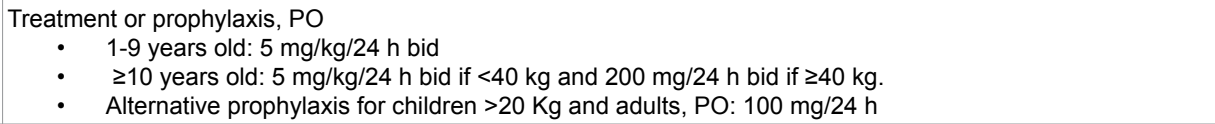 \\
\hline Rimantadine (Flumadine) & $\begin{array}{l}\text { Treatment ( } \geq 13 \text { years old), PO } 200 \mathrm{mg} / 24 \mathrm{~h} \text { bid } \\
\text { Prophylaxis (>1 year old), PO } \\
\begin{array}{l}-\quad 1-9 \text { years old: } 5 \mathrm{mg} / \mathrm{kg} / 24 \mathrm{~h} \\
-\quad \geq 10 \text { years old: } 5 \mathrm{mg} / \mathrm{kg} / 24 \mathrm{~h} \text { bid if }<40 \mathrm{~kg} \text { and } 200 \mathrm{mg} / 24 \mathrm{~h} \text { bid if } \geq 40 \mathrm{~kg}\end{array}\end{array}$ \\
\hline Oseltamivir (Tamiflu) ${ }^{c}$ & 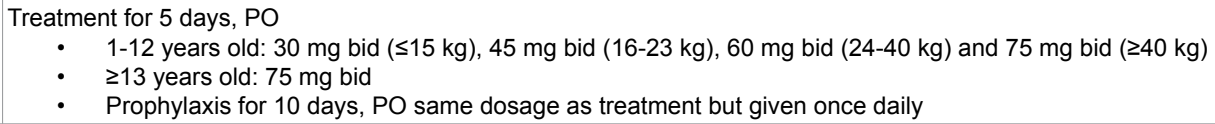 \\
\hline Zanamivir (Relenza) $^{d}$ & $\begin{array}{l}\text { Treatment for } 5 \text { days, inhaled } \\
\cdot \quad \geq 7 \text { years old: } 10 \mathrm{mg} \text { bid } \\
\text { Prophylaxis, inhaled } \\
\qquad \quad \geq 5 \text { years old: } 10 \mathrm{mg} / 24 \mathrm{~h} \text { once daily for } 28 \text { days (community outbreaks) or } 10 \text { days (household setting) }\end{array}$ \\
\hline
\end{tabular}

aEndo Pharmaceuticals, 100 Painters Drive, Chadds Ford, PA USA

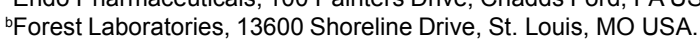

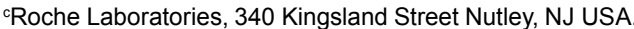

¿GlaxoSmithKline Pharmaceuticals, 5 Moore Drive, PO Box 13398 Research Triangle Park, NC USA.

Table 2: Recommended antiviral regimens for influenza infection.

\footnotetext{
- $5 \mathrm{mg} / \mathrm{Kg} /$ dose IV once weekly $\mathrm{x} 3$, followed by $5 \mathrm{mg} / \mathrm{Kg} / \mathrm{dose}$

$\begin{array}{ll}\text { Cidofovir (Vistide) } & \text { IV once every } 2 \text { weeks. } \\ \text { - Administer oral probenecid } 1-1,25 \mathrm{~g} / \mathrm{m}^{2} / \text { dose, } 3 \mathrm{hr} \text { prior to and } 1 \mathrm{hr} \text { and } 8 \mathrm{hr} \text { after each dose of cidofovir. }\end{array}$

- Administer IV normal saline x3 maintenance fluid, $1 \mathrm{hr}$ prior to and $1 \mathrm{hr}$ after cidofovir, followed by $\mathrm{x} 2$ maintenance fluid for an additional $2 \mathrm{hr}$

aGilead Sciences, 333 Lakeside Drive Foster City, CA USA.
}

Table 3: Recommended cidofovir regimen for adenoviral infection. 
Adoptive cellular immunotherapy using either donor adenovirusspecific $\mathrm{T}$ cells or $\mathrm{T}$ cells depleted from alloreactive cells offer new therapeutic options [27]. Future fields of research include the efficacy of combination antivirals, as to avoid the emergence of resistance and also to minimize adverse effects through the decrease of the required dosages of the respective agents $[10,11]$. Importantly, newer, so-called broad spectrum antivirals such as pyrimidine biosynthensis inhibitors or dsRNA activated caspase oligomerizers promise to treat a wide array of DNA and RNA viruses $[43,44]$.

\section{Prevention and Control Strategies}

Vaccination provides an established preventive method for influenza infection. The annual administration of the trivalent inactivated subunit vaccine, which contains two influenza A and one influenza B strains is recommended for children with cancer [11]. Unfortunately actual vaccination rates are still extremely low (9\%31\%) [45]. Among children with ALL vaccinated during re-induction, a seroresponse of $24 \%-60 \%$, and protective response rates of $57 \%-85 \%$ have been reported [46]. The protective antibody titres, although lower than in healthy children were considered satisfactory. In a recent study in children with solid tumors, the protective response rates were $60 \%$ $84 \%$, suggesting similar immunogenicity with leukemic children [47]. Overall, influenza vaccination in children receiving chemotherapy is strongly supported, since antibody titres are adequate and there are no safety issues. However, seroresponse rates vary according to chemotherapy phase and lymphocyte count. Although, new adjuvanted influenza vaccines are being evaluated in clinical trials, to prevent health-care related influenza spread, special emphasis must be placed on the promotion of yearly hospital staff vaccination $[45,48]$. Moreover, caregivers and family members in close contact with children with cancer should be educated on respiratory infections and "cocooning", i.e. the indirect protection of the susceptible person through their own vaccination [5]. Currently there are numerous studies for the development of commercial vaccines against RSV and adenovirus [11].

Moreover, general control measures for respiratory viruses include droplet and contact precautions (Table 1). These involve placing the infected patient in a single room or in the circumstance of multiple cases by the same pathogen, cohorting of the infected patients. Persons entering the room, including the health-care personnel, should wear a gown, gloves, mask, and eye protection. In the majority, a surgical mask is appropriate but for more contagious viruses, such as influenza H1N1 a fit-tested N95 mask is required. For more virulent viruses, negative pressure isolation is suggested $[36,49]$. Additionally to that, in the event of an outbreak, measures reducing effectively nosocomial transmission and increasing patient safety include: discharge of patients admitted for investigation or elective procedures, daily respiratory symptoms screening of staff, prompt sick-leave for staff exhibiting symptoms and discontinuation of transplantations as well as reduction of outpatient appointments for HSCT patients [11]. In the case of adenovirus infection, monitoring, similar to cytomegalovirus, prior and at specific intervals post HSCT may permit early detection in certain high-risk settings. Initiation and adherence to these control measures has been shown to cause significant reduction in the nosocomial spread of respiratory infections and increase patient safety at several oncology centres [10].

\section{Conclusions - Gaps in Knowledge}

Pediatric studies have often focused on a particular virus or a specific underlying malignancy making the overall assessment of the epidemiology of respiratory viral infections arduous. More studies are needed in order to define the true impact of these infections on mortality and morbidity of paediatric oncology patients, to better delineate therapeutic options and to identify ways of preventing such infections in these patients. Furthermore, few studies have utilized the latest -more sensitive and accurate-methods for viral detection. As a result there is a paucity of data regarding the epidemiology of recently emerged viral pathogens such as hMPV, bocavirus that play an important role in immunocompromised children $[2,9,11]$. Furthermore, it is hypothesized but not studied, that viral-bacterial co-infections are more frequent in immunocompromised patients compared to healthy subjects and are associated with increased clinical severity of respiratory infections due to impaired immune system and /or extensive pre-exposure to antibiotics. Moreover, it is difficult to compare incidence and disease burden between immunocompetent children and pediatric oncology patients since there have not been any studies evaluating URIs during the same period among these different cohorts. Recently, in a study involving immunocompetent children viral co-infection was a major factor associated with hospitalization [50]. This is in accordance with the aforementioned data since among pediatric oncology patients co-infections are associated with disease burden. Finally and most importantly, previous studies have focused on mortality as an outcome, while the issue of delays in chemotherapy due to respiratory viral infections and their potential association with patients' survival, has not been satisfactorily addressed to date.

\section{References}

1. Bailey LC, Reilly AF, Rheingold SR (2009) Infections in pediatric patients with hematologic malignancies. Semin Hematol 46: 313-324.

2. Kuypers J, Campbell AP, Cent A, Corey L, Boeckh M (2009) Comparison of conventional and molecular detection of respiratory viruses in hematopoietic cell transplant recipients. Transpl Infect Dis 11: 298-303.

3. Pizzo PA, Robichaud KJ, Wesley R, Commers JR (1982) Fever in the pediatric and young adult patient with cancer. A prospective study of 1001 episodes. Medicine (Baltimore) 61: 153-165.

4. Renaud C, Campbell AP (2011) Changing epidemiology of respiratory vira infections in hematopoietic cell transplant recipients and solid organ transplant recipients. Curr Opin Infect Dis 24: 333-343.

5. Fisher BT, Alexander S, Dvorak CC, Zaoutis TE, Zerr DM, et al. (2012) Epidemiology and potential preventative measures for viral infections in children with malignancy and those undergoing hematopoietic cell transplantation. Pediatr Blood Cancer 59: 11-15.

6. Katsimpardi K, Papadakis V, Pangalis A, Parcharidou A, Panagiotou JP, et al. (2006) Infections in a pediatric patient cohort with acute lymphoblastic leukemia during the entire course of treatment. Support Care Cancer 14: 277-284.

7. Luján-Zilbermann J, Benaim E, Tong X, Srivastava DK, Patrick CC, et al. (2001) Respiratory virus infections in pediatric hematopoietic stem cell transplantation Clin Infect Dis 33: 962-968.

8. Maltezou HC, Kafetzis DA, Abisaid D, Mantzouranis EC, Chan KW, et al. (2000) Viral infections in children undergoing hematopoietic stem cell transplant. Pediatr Infect Dis J 19: 307-312.

9. Whimbey E, Englund JA, Couch RB (1997) Community respiratory virus infections in immunocompromised patients with cancer. Am J Med 102: 10-18.

10. Hicks KL, Chemaly RF, Kontoyiannis DP (2003) Common community respiratory viruses in patients with cancer: more than just "common colds" Cancer 97: 2576-2587.

11. Kumar D, Humar A (2010) Respiratory viral infections in transplant and oncology patients. Infect Dis Clin North Am 24: 395-412.

12. Simon A, Schildgen O, Schuster F (2008) Viral infections in paediatric patients receiving conventional cancer chemotherapy. Arch Dis Child 93: 880-889.

13. Christensen MS, Nielsen LP, Hasle H (2005) Few but severe viral infections in children with cancer: a prospective RT-PCR and PCR-based 12-month study. Pediatr Blood Cancer 45: 945-951.

14. Koskenvuo M, Möttönen M, Rahiala J, Saarinen-Pihkala UM, Riikonen P, et al. (2008) Respiratory viral infections in children with leukemia. Pediatr Infect Dis J 27: $974-980$ 
Citation: Vliora C, Syridou G, Papaevangelou V (2014) Viral Respiratory Infections in Children Receiving Chemotherapy or Undergoing Stem Cell Transplantation. Clin Microbial 3: 137. doi:10.4172/2327-5073.1000137

15. Arola M, Ruuskanen O, Ziegler T, Salmi TT (1995) Respiratory virus infections during anticancer treatment in children. Pediatr Infect Dis J 14: 690-694.

16. Sung L, Gamis A, Alonzo TA, Buxton A, Britton K, et al. (2009) Infections and association with different intensity of chemotherapy in children with acute myeloid leukemia. Cancer 115: 1100-1108.

17. Salzer W, Dinndorf P, Dreyer ZA, Hilden J, Reaman GH (2009) Analysis of infectious complications in infants with acute lymphoblastic leukemia treated on the children's cancer group protocol 1953: A report from the Children's Oncology Group. J Pediatr Hematol Oncol 31: 398-405.

18. Sung L, Alonzo TA, Gerbing RB, Aplenc R, Lange BJ, et al. (2008) Respiratory syncytial virus infections in children with acute myeloid leukemia: a report from the Children's Oncology Group. Pediatr Blood Cancer 51: 784-786.

19. Kosmidi-Vasilatou E (2010) Contribution of the pediatrician in the care of a child with cancer. Proceedings of Practical Issues in Pediatric Hematology-Oncology.

20. Tasian SK, Park JR, Martin ET, Englund JA (2008) Influenza-associated morbidity in children with cancer. Pediatr Blood Cancer 50: 983-987.

21. Nichols WG, Corey L, Gooley T, Davis C, Boeckh M (2001) Parainfluenza virus infections after hematopoietic stem cell transplantation: risk factors, response to antiviral therapy, and effect on transplant outcome. Blood 98: 573-578.

22. Nichols WG, Guthrie KA, Corey L, Boeckh M (2004) Influenza infections after hematopoietic stem cell transplantation: risk factors, mortality, and the effect of antiviral therapy. Clin Infect Dis 39: 1300-1306.

23. Nichols WG, Gooley T, Boeckh M (2001) Community acquired respiratory syncytial virus and parainfluenza virus infections after hematopoietic stem cel transplantation: the Fred Hutchinson Cancer Research Center experience. Biol Blood Marrow Transplant 7(suppl): 11S-15S.

24. Lim do H, Lee J, Lee HG, Park BB, Peck KR, et al. (2006) Pulmonary complications after hematopoietic stem cell transplantation. J Korean Med Sci 21: 406-411.

25. Peck AJ, Englund JA, Kuypers J, Guthrie KA, Corey L, et al. (2007) Respiratory virus infection among hematopoietic cell transplant recipients: evidence for asymptomatic parainfluenza virus infection. Blood 110: 1681-1688.

26. Hale GA, Heslop HE, Krance RA, Brenner MA, Jayawardene D, et al. (1999) Adenovirus infection after pediatric bone marrow transplantation. Bone Marrow Transplant 23: 277-282.

27. van Tol MJ, Kroes AC, Schinkel J, Dinkelaar W, Claas EC, et al. (2005) Adenovirus infection in paediatric stem cell transplant recipients: increased risk in young children with a delayed immune recovery. Bone Marrow Transplant 36: 39-50.

28. Runde V, Ross S, Trenschel R, Lagemann E, Basu O, et al. (2001) Adenoviral infection after allogeneic stem cell transplantation (SCT): Report on 130 patients from a single SCT unit involved in a prospective multi center surveillance study. Bone Marrow Transplant 28: 51-57.

29. Cole PD, Suh JS, Onel K, Stiles J, Armstrong D, et al. (2001) Benign outcome of RSV infection in children with cancer. Med Pediatr Oncol 37: 24-29.

30. El-Mahallawy HA, Ibrahim MH, Shalaby L, Kandil A (2005) Community respiratory viruses as a cause of lower respiratory tract infections following suppressive chemotherapy in cancer patients. Journal of the Egyptian Nat Cancer Inst 17: 121-126.

31. El Saleeby CM, Somes GW, DeVincenzo JP, Gaur AH (2008) Risk factors for severe respiratory syncytial virus disease in children with cancer: the importance of lymphopenia and young age. Pediatrics 121: 235-243.

32. Mendoza Sánchez MC, Ruiz-Contreras J, Vivanco JL, Fernández-Carrión F, Baro Fernández M, et al. (2006) Respiratory virus infections in children with cancer or HIV infection. J Pediatr Hematol Oncol 28: 154-159.

33. Verdeguer A, de Heredia CD, González M, Martínez AM, Fernández-Navarro $\mathrm{JM}$, et al. (2011) Observational prospective study of viral infections in children undergoing allogeneic hematopoietic cell transplantation: a 3-year GETMON experience. Bone Marrow Transplant 46: 119-124.

34. Milano F, Campbell AP, Guthrie KA, Kuypers J, Englund JA, et al. (2010)
Human rhinovirus and coronavirus detection among allogeneic hematopoietic stem cell transplantation recipients. Blood 115: 2088-2094.

35. Piralla A, Percivalle E, Di Cesare-Merlone A, Locatelli F, Gerna G (2009) Multicluster nosocomial outbreak of parainfluenza virus type 3 infection in a pediatric oncohematology unit: a phylogenetic study. Haematologica 94: 833839

36. Pickering L, Baker C, Kimberlin D, Long S (2009) Red book: 2009 Report of the Committee on Infectious Diseases. (28th ed) American Academy of Pediatrics Elk Grove Village, Illinois.

37. Medeiros R, Rameix-Welti MA, Lorin V, Ribaud P, Manuguerra JC, et al. (2007) Failure of zanamivir therapy for pneumonia in a bone-marrow transplant recipient infected by a zanamivir-sensitive influenza $A(H 1 N 1)$ virus. Antivir Ther 12: $571-576$

38. Torres HA, Aguilera EA, Mattiuzzi GN, Cabanillas ME, Rohatgi N, et al. (2007) Characteristics and outcome of respiratory syncytial virus infection in patients with leukemia. Haematologica 92: 1216-1223.

39. Legrand F, Berrebi D, Houhou N, Freymuth F, Faye A, et al. (2001) Early diagnosis of adenovirus infection and treatment with cidofovir after bone marrow transplantation in children. Bone Marrow Transplant 27: 621-626.

40. Doan ML, Mallory GB, Kaplan SL, Dishop MK, Schecter MG, et al. (2007) Treatment of adenovirus pneumonia with cidofovir in pediatric lung transplant recipients. J Heart Lung Transplant 26: 883-889.

41. Yusuf U, Hale GA, Carr J, Gu Z, Benaim E, et al. (2006) Cidofovir for the treatment of adenoviral infection in pediatric hematopoietic stem cell transplant patients. Transplantation 81: 1398-1404.

42. Custer J, Rau R (2009) The Harriet Lane Handbook. (18thedn), Mosby Elsevier Philadelphia, USA.

43. Hoffmann HH, Kunz A, Simon VA, Palese P, Shaw ML (2011) Broad-spectrum antiviral that interferes with de novo pyrimidine biosynthesis. Proc Natl Acad Sci U S A 108: 5777-5782.

44. Rider TH, Zook CE, Boettcher TL, Wick ST, Pancoast JS, et al. (2011) Broadspectrum antiviral therapeutics. PLoS One 6: e22572.

45. Esposito S, Cecinati V, Russo FG, Principi N (2009) Influenza vaccination in children with cancer receiving chemotherapy. Hum Vaccin 5: 430-432.

46. Hsieh YC, Lu MY, Kao CL, Chiang BL, Lin DT, et al. (2002) Response to influenza vaccine in children with leukemia undergoing chemotherapy. J Formos Med Assoc 101: 700-704.

47. Bektas O, Karadeniz C, Oguz A, Berberoglu S, Yilmaz N, et al. (2007) Assessment of the immune response to trivalent split influenza vaccine in children with solid tumors. Pediatr Blood Cancer 49: 914-917.

48. Helms CM, Polgreen PM (2008) Should influenza immunisation be mandatory for healthcare workers? Yes. BMJ 337: a2142.

49. Siegel JD, Rhinehart E, Jackson M, Chiarello L; Health Care Infection Contro Practices Advisory Committee (2007) 2007 Guideline for Isolation Precautions: Preventing Transmission of Infectious Agents in Health Care Settings. Am J Infect Control 35: S65-164.

50. Kouni S, Karakitsos P, Chranioti A, Theodoridou M, Chrousos G, et al. (2013) Evaluation of viral co-infections in hospitalized and non-hospitalized children with respiratory infections using microarrays. Clin Microbiol Infect 19: 772-777. 